\title{
Découverte d'un nouvel inhibiteur de l'angiogenèse, I'angiostatine. Implications pour la biologie et la thérapie des métastases
}

La croissance d'une tumeur solide et la dissémination métastatique dépendent de la formation de nouveaux vaisseaux sanguins, ou angiogenèse. Ces vaisseaux irriguent la tumeur primitive et fournissent aux cellules tumorales une voie pour s'échapper du site primitif et aller établir des métastases [1]. A côté des interactions avec le stroma qui constitue l'environnement immédiat des cellules cancéreuses, la tumeur primitive est aussi susceptible d'interagir à distance avec les micrométastases. Les chercheurs du laboratoire de Judah Folkman à Boston (MA, USA) ont analysé un modèle murin dans lequel la présence de la tumeur primitive inhibe presque complètement la croissance des métastases. Dans un article récent [2], ils décrivent la purification et le séquençage d'un polypeptide nommé angiostatine qui serait responsable de cette inhibition.

Des cellules de carcinome pulmonaire (Lewis lung carcinoma ou 3LL) furent implantées sous la peau, dans le dos de souris syngéniques ou de souris immunodéficientes. Dès le troisième jour, des tumeurs visibles étaient observées ; elles atteignirent la taille de 800 à $2000 \mathrm{~mm}^{3}$ en quinze jours. Les souris furent alors divisées en deux groupes : dans le premier groupe fut réalisée l'exérèse chirurgicale des tumeurs, tandis que dans le second groupe, ou groupe témoin, les tumeurs furent laissées en place, après une incision de la peau et une manipulation de ces tumeurs qui mimait le geste chirurgical d'excision tumorale. L'ablation de la tumeur fut suivie d'une croissance rapide des métastases pulmonaires ; 13 à 21 jours après cette ablation, le nombre poumons était multiplié par dix et le poids des poumons par quatre, par comparaison avec les souris témoins dont la tumeur primitive était restée en place. L'observation histologique révéla des micrométastases dans les poumons des souris témoins, et une intense vascularisation dans les métastases développées dans les poumons des souris auxquelles on avait ôté la tumeur primitive. Dans ce modèle, la présence d'une tumeur primitive semblait donc inhiber la néovascularisation et la croissance des métastases, sans empêcher leur dissémination. Cette activité anti-angiogénique pouvait aussi s'exercer sur d'autres sites, puisqu'un implant de bFGF (basic fibroblast growth factor), un puissant facteur angiogénique [2], était incapable d'induire l'angiogenèse dans la cornée de souris porteuses d'une tumeur primitive d'au moins $2000 \mathrm{~mm}^{3}$.

Purification et isolement d'un nouvel inhibiteur de l'angiogenèse qui bloque la croissance des métastases

La purification de cette activité inhibitrice fut facilitée par la détection dans le sérum et l'urine des souris portant la tumeur primitive d'une activité supprimant la croissance des métastases. Le sérum et l'urine de ces souris étaient aussi capables d'inhiber, in vitro, la prolifération des cellules endothéliales de façon dépendante de la dose. Cette inhibition était spécifique puisque aucun effet reproductible ne fut observé sur la prolifération de douze autres types de cellules. Cette activité inhibitrice circulante fut associée à une protéine de $38 \mathrm{kDa}$, purifiée à la fois dans le sérum et dans l'urine des souris por- tant des tumeurs primitives ; le microséquençage révéla une identité de $98 \%$ avec un fragment interne du plasminogène de souris qui inclut quatre de ses cinq triples boucles (ou kringles) (figure 1). Cette protéine fut nommée angiostatine.

Des fragments du plasminogène humain, engendrés par digestion protéolytique limitée par l'élastase, sont capables d'inhiber l'angiogenèse dans la membrane chorioallantoïde de poulet et d'inhiber la prolifération des cellules endothéliales in vitro, mais le plasminogène entier n'inhibe pas la prolifération des cellules endothéliales. Une situation analogue est rencontrée avec la prolactine et la fibronectine, dont seuls des fragments possèdent une activité inhibitrice spécifique sur les cellules endothéliales. L'efficacité de l'angiostatine est comparable à celle d'autres inhibiteurs physiologiques de l'angiogenèse, comme la thrombospondine-1 et les fragments protéolytiques dérivés de la thrombospondine. La thrombospondine-1 peut être sécrétée par les cellules tumorales elles-mêmes et, dans un système expérimental, cette sécrétion diminue lorsque les cellules tumorales basculent vers un phénotype angiogénique. Les mécanismes qui contrôlent la production d'angiostatine ne sont pas encore élucidés. L'activité angiostatine dans le sérum des souris est associée à la présence d'une tumeur primitive : il sera important de déterminer si les cellules 3LL utilisées produisent le plasminogène ou une activité élastase capable de libérer l'angiostatine à partir du plasminogène. Il est intéressant de noter qu'un autre fragment du plasminogène, incluant uniquement la 
quatrième boucle, stimule la prolifération des cellules endothéliales, et que la thrombospondine-1 interagit spécifiquement avec le plasminogène (figure 1). Le plasminogène et la thrombospondine sont donc bien des protéines multifonctionnelles qui participent au réseau de régulations contrôlant l'angiogenèse.

Plusieurs hypothèses pouvaient être émises pour expliquer l'inhibition de la croissance des métastases par la tumeur primitive dans le système expérimental décrit : (1) une déplétion par la tumeur primitive des éléments nutritifs disponibles ou (2) la production par la tumeur primitive de facteurs qui bloqueraient la prolifération des cellules métastatiques. La découverte de l'angiostatine suggère que cette inhibition des métastases par la tumeur primitive est relayée, en partie au moins, par une inhibition de l'angiogenèse au niveau de ces métastases. En effet, après abla- tion de la tumeur primitive, la croissance des métastases peut être inhibée par injection intrapéritonéale de sérum ou d'urine provenant de souris porteuses d'une tumeur primitive ; la même inhibition est observée après l'injection de fragments du plasminogène humain. Si l'angiostatine est soustraite de l'urine par passage sur une colonne de Sépharose couplée à un anticorps dirigé contre le plasminogène humain, les effets antimétastatiques in vivo et antiprolifératifs in vitro ne sont plus observés.

\section{Les limites du modèle expérimental et les implications thérapeutiques}

Pour évaluer la portée de ce résultat, il faut d'abord considérer de plus près le modèle expérimental utilisé. Les auteurs écrivent que les souris sont opérées de leur tumeur primitive alors que le volume de cette tumeur est de $1500 \mathrm{~mm}^{3}$. C'est déjà

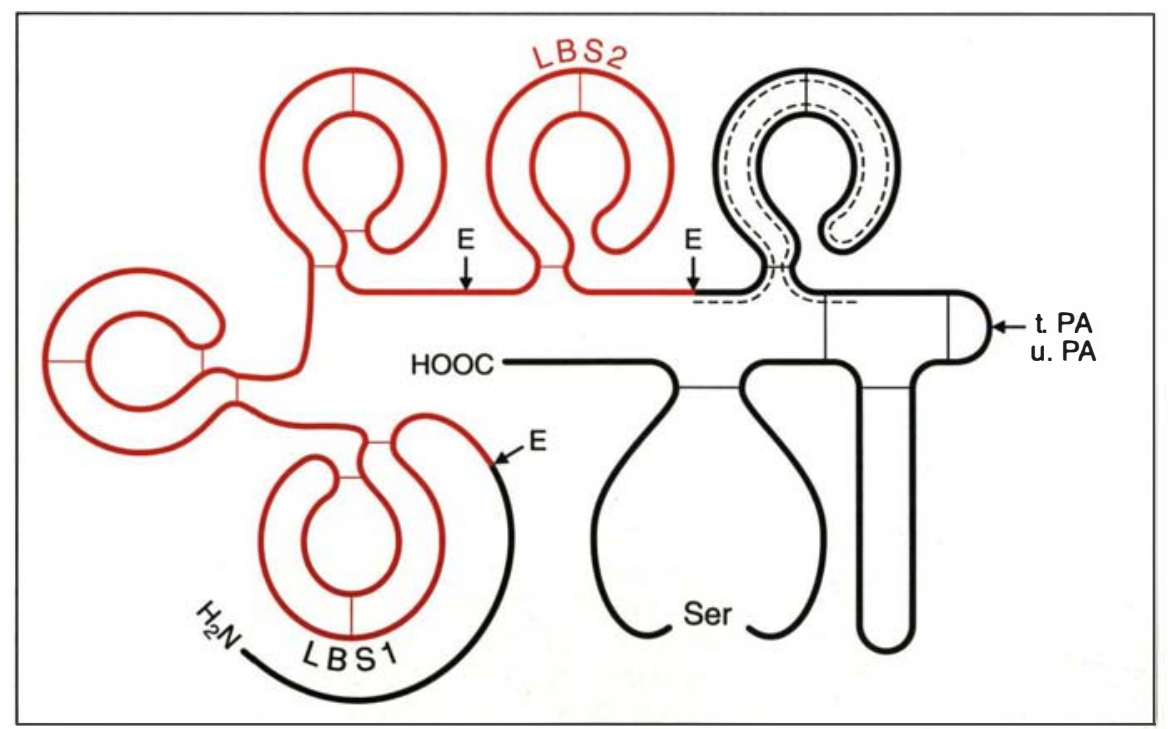

Figure 1. Angiostatine et plasminogène. Structure de la molécule de plasminogène, avec les cinq triples hélices ou kringles, les sites de fixation de la lysine (LBS 1 et 2), le site catalytique (ser), les sites de coupure protéolytique par l'élastase (E) et par les activateurs du plasminogène, UPA et IPA. Le domaine d'interaction avec la thrombospondine-1 est souligné par des pointillés noirs. La chaîne polypeptidique correspondant à l'angiostatine est indiquée en rouge. D'après le groupe de Judah Folkman, le fragment protéolytique correspondant au second site de fixation de la lysine (LBS2) stimule la prolifération des cellules endothéliales.

$\mathrm{m} / \mathrm{s} n^{\circ} 2$, vol. 11 , fárier 95 une taille importante, qui correspond à 14 jours d'évolution. Les souris témoins, non opérées, doivent survivre jusqu'à 14 ou 21 jours de plus : pour la collecte d'urine, la taille de tumeurs atteint $3500 \mathrm{~mm}^{3}$ ! Ce modèle semble contestable pour des raisons d'éthique animale, et on peut s'étonner que la publication de ces résultats n'ait pas été refusée ! De plus, il est vraisemblable que ces tumeurs, qui représentent une masse considérable par rapport à la souris, se nécrosent et libèrent une grande quantité de produits de nécrose. Ces produits sont susceptibles d'inhiber directement la prolifération des cellules métastatiques.

Il ne faut pas non plus oublier les nombreux modèles expérimentaux de tumeurs dans lesquels on n'observe pas d'inhibition de la croissance des métastases ou dans lesquels cette croissance est inhibée par des mécanismes différents. Un sous-clone des cellules 3LL est décrit dans l'article, qui est incapable d'inhiber la croissance des métastases. Les auteurs écrivent que le sérum des souris porteuses de tumeur ne présente pas d'activité angiostatine ; mais ils ne précisent pas la taille qu'atteint la tumeur primitive avec ce variant.

Comme l'écrit Isaiah Fidler (Houston, TX, USA), dans un article de revue [3] publié dans le même numéro de Cell, il faut souligner ici que la découverte de l'angiostatine ne signifie absolument pas que les chirurgiens ne doivent pas opérer les tumeurs primitives par crainte de voir s'accélérer la croissance de micro-métastases non détectées. On peut, certes, comme le fait Judah Folkman dans l'introduction de son article, aligner une bibliographie consistante décrivant une croissance accélérée des métastases après ablation de la tumeur primitive. Mais le nombre de cas recensés est extrêmement faible par rapport au nombre de patients opérés chez lesquels ce phénomène n'a pas été observé. Parce que le risque de métastases est directement proportionnel au nombre de cellules métastatiques qui entrent dans la circulation, il semble préférable de pratiquer l'ablation de la tumeur primitive aussi tôt que possible afin de préve- 
nir cette dissémination. Dans le traitement de la plupart des tumeurs solides, seule la chirurgie est une thérapie curative. On sait que des métastases peuvent se dévoiler ultérieurement, en particulier dans le cas du cancer du sein pour lequel la courbe de survie des malades après chirurgie et traitement radio ou chimiothérapique s'abaisse de façon constante. Chez ces sujets sans maladie apparente, des métastases peuvent donc survenir tardivement. Mais il apparaît clairement que, dans ce cas, l'acte chirurgical n'accélère pas la croissance des métastases.

Il est vraisemblable que des travaux futurs détermineront quelles tumeurs humaines sont associées à une circulation élevée d'angiostatine. On peut imaginer que, pour ces patients, l'ablation de la tumeur primitive soit suivie d'une thérapie adjuvante ciblée contre l'angiogenèse, avec l'angiostatine ou d'autres inhibiteurs.

Dans des modèles expérimentaux, plusieurs stratégies ont été développées avec succès pour inhiber l'angiogenèse tumorale. Ainsi, l'administration systémique d'AGM 1470 [4], d'anticorps contre le bFGF, le VEGF (vascular endothelial growth factor) ou l'angiogénine un ou deux jours après l'implantation de cellules concéreuses sous la peau inhibent la croissance tumorale en bloquant l'angiogenèse. Cependant, avant d'envisager l'utilisation d'une telle démarche thérapeutique chez l'homme, il est intéressant de rappeler qu'elle peut être contradictoire avec le désir d'augmenter l'irrigation de la tumeur pour améliorer l'accès des médicaments en chimiothérapie, ou pour améliorer l'oxygénation des cellules cancéreuses dans le cas des radiothérapies. Il est aussi important de considérer que c'est l'anoxie qui tue les cellules cancéreuses, tandis que l'hypoxie a des effets adverses : induction de la production de facteurs angiogéniques, sélection de cellules résistantes et aggravation de la malignité des cellules cancéreuses. Les analyses in vitro et l'étude des modèles expérimentaux chez la souris permettent d'identifier les acteurs cellulaires et moléculaires de l'angio- genèse tumorale. Une analyse systémique qui permette d'évaluer l'importance et la quantité de chacun de ces composants, leur cinétique d'apparition et leurs interactions paraît indispensable avant d'envisager une utilisation thérapeutique chez l'homme.

B.V. M.F.P.

1. Vandenbunder B, Fafeur V, Wernert N, Stéhe lin D. Analyse moléculaire de l'angiogenèse tumorale. médecine/sciences $1994 ; 10$ : 516-27. 2. O'Reilly MS, Holmgren L, Shing Y, Chen C, Rosenthal RA, Moses M, Lane WS, Cao Y, Sage EH Folkman J. Angiostatin : a novel angiogenesis inhibitor that mediates the suppression of metastases by a Lewis lung carcinoma. Cell $1994 ; 79: 315-28$. 3. Fidler IJ, Ellis LM. The implication of angioge nesis for the biology and therapy of cancer metastasis. Cell $1994 ; 79$ : 185-8.

4. Ingber D, Fujita T, Kishimoto S, Sudo K, Kanamaru T, Brem H, Folkman J. Synthetic analogues

\section{BRÈVES}

Neuropeptides : la peau de la grenouille n'a pas dit son demier mot. Les glandes à poison de la peau des amphibiens constituent une source exceptionnellement riche de peptides biologiquement actifs. L'abondance des peptides synthétisés y est telle que l'on peut souvent, à partir de la peau d'un seul animal, purifier suffisamment de matériel pour en déterminer la séquence. A titre d'exemple, la peau de certains amphibiens anoures contient environ $1 \mathrm{mg}$ de thyrolibérine (TRH), quantité comparable à celle qu'avait isolée l'équipe de Roger Guillemin à partir de quelque 500000 hypothalamus de mouton [1]. A ce jour, plus de 110 peptides différents ont été caractérisés à partir d'extraits de peau d'amphibiens, en particulier par l'équipe italienne de Vittorio Erspamer [2]. La plupart de ces peptides appartiennent à des familles de polypeptides également exprimés dans le cerveau ou le tube digestif des mammifères : tachykinines, peptides opiacés, gastrine/cholécystokinine, bombésine/gastrin releasing peptide... Toutefois, à ce jour, aucun peptide de la famille du neuropeptide tyrosine (NPY) n'avait été identifié dans la peau des amphibiens. Deux équipes françaises (Institut Jacques Monod, Paris et INSERM U 413, Rouen) ont isolé récemment un nouveau peptide à partir de la peau d'une grenouille arboricole sud-américaine Phyllomedusa bicolor [3]. Il s'agit d'un peptide de 36 acides aminés qui présente une forte analogie de séquence avec le peptide tyrosine-tyrosine (PYY), un peptide de la famille du NPY. Le nouveau peptide, appelé skin peptide tyrosine-tyrosine (SPYY) présente une activité mélanostatine (inhibition de la sécrétion de l'hormone mélanotrope par les cellules du lobe intermédiaire de l'hypophyse) comparable à celle du NPY [4]. La présence dans la peau de deux peptides hypophysiotropes qui contrôlent la sécrétion de l'hormone mélanotrope (TRH et SPYY) suggère l'existence d'une nouvelle boucle de régulation neuroendocrinienne entre le lobe intermédiaire de l'hypophyse et la peau chez les amphibiens.

[1. Burgus R, et al. Nature 1970 ; 350 : 498-500.]

[2. Erspamer V. Trends Neurosci 1983 ; 6 : 200-1.]

[3. Mor A, et al. Proc Natl Acad Sci USA 1994 ; 91 : 10295-9.]

[4. Chartrel N, et al. Proc Natl Acad Sci USA 1991 ; 88 : 3862-6.] 